\title{
Accuracy of Intraoperative Frozen Section in the Diagnosis of Ovarian Neoplasms
}

\author{
Amany M. Abdelghany, Essa M. Arafa, Nadia M. Madkour, Wael S. Nossair*, \\ Ekramy A. Mohamed, Walid A. Abdelsalam, Amira A. Salem \\ Obstetrics \& Gynecology Department, Faculty of Medicine, Zagazig University, Zagazig, Egypt \\ Email: ${ }^{*}$ wsnossair@gmail.com
}

Received 24 September 2014; revised 30 October 2014; accepted 10 November 2014

Academic Editor: Yasam Kemal Akpak, Ankara Mevki Military Hospital, Turkey

Copyright (C) 2015 by authors and Scientific Research Publishing Inc.

This work is licensed under the Creative Commons Attribution International License (CC BY). http://creativecommons.org/licenses/by/4.0/

(c) (i) Open Access

\section{Abstract}

Objective: The aim of the work is to evaluate the accuracy of intraoperative frozen section in the diagnosis of ovarian neoplasms in Zagazig University. Design: A prospective cross sectional cohort study. Method: This study was performed between March 2011 and March 2012, on 50 patients presented with ovarian mass. Gross examination of the tumor removed was done by inspection and palpation. The specimen was then cut with a sharp knife into two halves. The most appropriate area thought to be representative of lesion was chosen. The number of sections frozen was depended on the type and size of the tumor. Seven to eight $\mu \mathrm{m}$ sections were obtained and stained with hematoxylin-eosin. The specimens were then fixed in formalin. Paraffin blocks of the sections were processed in the routine way and sections were stained with hematoxylin and eosin ( $\mathrm{H}$ and E). The diagnosis obtained by intraoperative frozen section based on cellularity and cell morphology was compared with final histopathological diagnosis in terms of diagnostic sensitivity, to differentiate between benign and malignant lesions. Assessment of the overall accuracy of the intraoperative diagnosis was classified as concordant or discordant. Results: There was no statisticaly significant differencre in the studied patients as regard the clinical data, macroscopic and intraoperative picture, while there was statisticaly significat association as regard the laterality of the ovarian masses. The validity of frozen section in the diagnosis of benign tumour was $100 \%$ with $100 \%$ accuracy, specificity, positive predictive value, negative predictive value, while sensitivity $\&$ negative prediction for borderline tumour and specificity $\&$ positive prediction of malignant tumour were $100 \%$, specifecity for borderline tumours was $95 \%$ while the positive predictive value was $33.3 \%$ with $\mathbf{9 6 \%}$ accuracy for both malignant and borderline tumours. Conclusion: Intraoperative frozen section is accurate for rapid diagnosis of ovarian tumors. It can help surgeons avoid under-treatment or overtreatment of patients. Our study was designed prospectively

\footnotetext{
${ }^{*}$ Corresponding author.
}

How to cite this paper: Abdelghany, A.M., Arafa, E.M., Madkour, N.M., Nossair, W.S., Mohamed, E.A., Abdelsalam, W.A. and Salem, A.A. (2015) Accuracy of Intraoperative Frozen Section in the Diagnosis of Ovarian Neoplasms. Open Journal of Obstetrics and Gynecology, 5, 14-22. http://dx.doi.org/10.4236/ojog.2015.51003 
using a small number of patients. The door is open to larger studies using a larger number of patients to be performed in order to substantiate our results.

\title{
Keywords
}

\author{
Intraoperative, Frozen Section, Ovarian Neoplasms
}

\section{Introduction}

Ovarian neoplasms are a heterogeneous group of benign, borderline and malignant tumors of epithelial, stromal and germ-cell origin [1]. Ovarian cancer is the fifth commonest cancer in women in the UK after breast, colorectal, lung and uterus. Approximately 6700 new cases of ovarian cancer were diagnosed every year in United Kingdom between 2004 and 2007 accounting for approximately 1 in 20 cases of cancer in women [2]. Histopathology of a paraffin section remains the ultimate gold standard in tissue diagnosis [3].

Rapid intra-operative diagnosis of the nature of ovarian tumors is both interesting and challenging for effective planning of the surgical management of these tumors, particularly in a young woman as it can avoid unnecessary removal of contralateral ovary and helps preserve fertility [4]. Intra-operative diagnosis of ovarian lesions can be achieved by a number of cytological techniques, including imprint and scrape cytology, and intraoperative fine-needle aspiration cytology (FNAC). These methods are less accurate as compared to frozen section (FS) [5].

Intraoperative FS for use in diagnosis of ovarian tumors is of great value. In some cases, it can help surgeons avoid under-treatment or overtreatment of patients. FS is indicated to ensure that the tissue sampled adequate for diagnosis, to determine the nature of the disease process, to plan ancillary studies, to determine tumor spread, and to assess margins [6] [7]

The aim of the work is to evaluate the accuracy of intraoperative frozen section in the diagnosis of ovarian neoplasms in Zagazig University.

\section{Patients and Methods}

This study was performed between March 2011 and March 2012, on 50 patients presented with ovarian mass recruited from the gynecology outpatient clinic. Patients with the following criteria were included: 1) clinically benign looking tumors (on preoperative radiology or intraoperative inspection) with raised CA-125; 2) adnexal mass in a patient with a past history of malignancy at another site; iii) young patients with ovarian neoplasms in whom fertility sparing surgery was planned; and iv) adnexal mass in any perimenopausal women.

The following was done for all selected patients: full history; full clinical examination and measurement of serum CA 125. The preoperative data studded were patient age, rapid weight loss, clinical examination, available imaging studies either ultrasonography, Computerized Tomography or both, level of the available tumor marker such as serum CA 125.

Patients with benign-appearing tumors at surgery underwent either cystectomy or salpingo-oophorectomy, depending on patient and tumor characteristics. Patients with suspicious ovarian tumors underwent salpingooophorectomy. The intraoperative findings studded were presence of ascitis, adhesions to the adjacent structures, bilaterality, tumor implants on peritoneal surface, lymph node enlargement and gross picture of the tumor (e.g. tumor diameters, consistency, outer surface, cut surface, presence of necrotic areas and papillary structures within the cyst).

The unfixed fresh specimen of the tumor was immediately delivered to Pathology Department-Faculty of Medicine-Zagazig University - with all the clinical details of the patient for evaluation. Gross examination of the tumor removed was done by inspection and palpation, The specimen was then cut with a sharp knife into two halves. The cut surface was wiped off the excess blood, if present, with the help of a filter paper. Again, reinspection and repalpation of the tumor was done.

The most appropriate area thought to be representative of lesion was chosen. One to four frozen sections per case were taken from different representative areas. The number of frozen sections was depended on the type and size of the tumor. Seven to eight $\mu \mathrm{m}$ sections were obtained and stained with hematoxylin-eosin. 

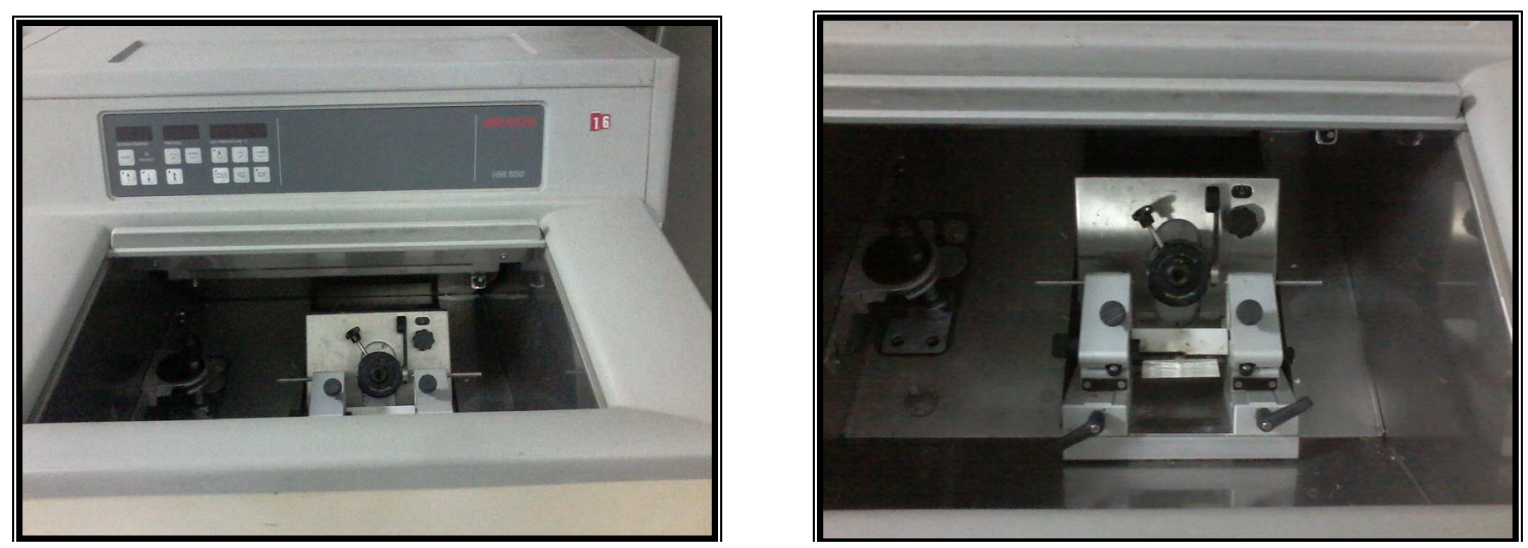

The specimens were then fixed in formalin. Paraffin blocks of the sections were processed in the routine way and sections were stained with hematoxylin and eosin ( $\mathrm{H}$ and $\mathrm{E}$ ). All the sections were studied microscopically under low and high power and categorized according to the status of malignancy into benign, borderline and malignant tumor.

The diagnosis obtained by intraoperative frozen section based on cellularity and cell morphology was compared with final histopathological diagnosis in terms of diagnostic sensitivity, to differentiate between benign and malignant lesions. Assessment of the overall accuracy of the intraoperative diagnosis was classified as follows:

- Concordant. In these cases, the diagnosis was correct with regard to the major tumor category (benign, borderline, or malignant) and, in the case of malignant neoplasms, to tumor origin (primary vs metastatic).

- Discordant. These were cases in which incorrect or equivocal assessment could have adversely affected intraoperative management, including the failure to identify the principle diagnostic category of surface epithelial tumors (benign, borderline, or malignant) or inaccuracy regarding the origin of malignant neoplasms (primary vs metastatic). Cases in which the major histological category of primary ovarian malignancy (carcinoma, sex cord stromal tumor, and germ cell tumor) was not recognized were also considered discordant because the optimal operative management of these cases may differ, particularly in the younger age group.

Statistical analysis was performed using STATA 11.0 (College Station, Texas, USA). Data were presented as a number (\%) or mean \pm standard deviation (SD)/median (range). The differences in Mean/Median values were measured by using Student's t-test (normal data distribution)/Wilcoxon Ranksum test (for categorical variables). $\mathrm{p}<0.05$ was considered to be statistically significant.

The study was approved by Zagazig University Medical Research Council Laboratories Joint Ethics Committee. All participants signed an informed consent. Rigorous confidentiality was maintained.

\section{Results}

The study was performed on 50 (age ranging between 11 and 77 years) patients presented with ovarian mass. Table 1 shows the features of the studied patients in relation to the type of ovarian masses. Different types of ovarian masses (benign, borderline and malignant) show statistical significant correlation with the age of the patient while there is no significant association between parity and different types of ovarian masses. Table 2 shows the association between different types of ovarian masses and clinical data, macroscopic \& intraoperative picture, and laterality of ovarian masses. There was no statistically significant difference in the studied patients as regard the clinical data, macroscopic and intraoperative picture, while there was statistically significant association as regard the laterality of the ovarian masses. Table 3 shows the histological types of the tumors according to the results of paraffin sections, their number and percentage among patients under the study. Table 4 and Table 5 show the validity of frozen section in the diagnosis of ovarian masses. The validity of frozen section in the diagnosis of benign tumour was $100 \%$ with $100 \%$ accuracy, specificity, positive predictive value, negative predictive value, while sensitivity \& negative prediction for borderline tumour and specificity \& positive prediction of malignant tumour were $100 \%$, specificity for borderline tumours was $95 \%$ while the positive predictive value was $33.3 \%$ with $96 \%$ accuracy for both malignant and borderline tumours. 
Table 1. Features of the studied patients (Age and parity) in relation to the type of ovarian masses ((benign, borderline and malignant).

\begin{tabular}{|c|c|c|c|c|c|}
\hline & $\begin{array}{c}\text { Benign } \\
(n=29)\end{array}$ & $\begin{array}{c}\text { Borderline } \\
(\mathrm{n}=1)\end{array}$ & $\begin{array}{c}\text { Malignant } \\
(\mathrm{n}=20)\end{array}$ & $\mathbf{F}$ & $\mathbf{p}$ \\
\hline \multicolumn{6}{|l|}{ Age (years) } \\
\hline Mean \pm SD & $36.5 \pm 17.7$ & $43 \pm 0$ & $48.9 \pm 12.7$ & 3.57 & 0.03 \\
\hline Range & $11-70$ & & $19-77$ & & \\
\hline \multicolumn{6}{|l|}{ Parity } \\
\hline $\mathbf{0}$ & $13(44.8 \%)$ & $1(100 \%)$ & $6(30 \%)$ & & \\
\hline $1-3$ & 7 (24.1\%) & $0(0 \%)$ & 10 (50\%) & $X^{2}=5.06$ & 0.28 \\
\hline$>3$ & 9 (31\%) & $0(0 \%)$ & $4(20 \%)$ & & \\
\hline
\end{tabular}

Table 2. Association between different types of ovarian masses and clinical data, macroscopic \& intraoperative picture, and laterality of ovarian masses.

\begin{tabular}{|c|c|c|c|c|c|c|c|c|}
\hline & \multicolumn{2}{|c|}{ Benign } & \multicolumn{2}{|c|}{ Borderline } & \multicolumn{2}{|c|}{ Malignant } & \multirow{2}{*}{$\mathbf{X}^{2}$} & \multirow{2}{*}{$\mathbf{p}$} \\
\hline & No & $\%$ & No & $\%$ & No & $\%$ & & \\
\hline Clinical & 21 & 42.0 & 0 & 0.0 & 29 & 58.0 & \multirow{2}{*}{0.04} & \multirow{2}{*}{0.8} \\
\hline Macroscopic and intraoperative picture & 20 & 40.0 & 0 & 0.0 & 30 & 60.0 & & \\
\hline \multicolumn{9}{|l|}{ Laterality } \\
\hline Unilateral & 20 & 69.0 & 1 & 100.0 & 6 & 30.0 & \multirow{2}{*}{8.1} & \multirow{2}{*}{0.017} \\
\hline Bilateral & 9 & 31.0 & 0 & 0.0 & 14 & 70.0 & & \\
\hline
\end{tabular}

Table 3. The results of paraffin sections.

\begin{tabular}{|c|c|c|c|}
\hline Histologic type & Number of cases & $(\%)$ & N B: \\
\hline \multicolumn{4}{|l|}{ A. Epithelial tumors: } \\
\hline \multicolumn{4}{|l|}{ *Benign: } \\
\hline \multirow[t]{2}{*}{ Serous } & 8 cases & $16 \%$ & ${ }^{*} 3 / 8$ complicated \\
\hline & & & *5/8 noncomplicated \\
\hline \multirow[t]{2}{*}{ Mucinous } & 3 cases & $6 \%$ & *1/8 bilateral \\
\hline & & & ${ }^{*} 1 / 3$ complicated \\
\hline \multirow[t]{3}{*}{ Brenner. } & & & ${ }^{*} 2 / 3$ non complicated \\
\hline & 1 case & $2 \%$ & ${ }^{*} 1 / 3$ bilateral \\
\hline & & & Unilateral \\
\hline \multicolumn{4}{|l|}{ "Malignant. } \\
\hline Serouscyst adenoarcinoma & 12 case & $24 \%$ & *4/12 Serouscyst adenoarcinoma, 3/4 bilateral. \\
\hline & & & *8/12 papillary Serouscyst adenoarcinoma,6/8 bilat \\
\hline Endometrioid adenocarinoma & 6 cases & $12 \%$ & ${ }^{*} 3 / 6$ bilateral \\
\hline \multicolumn{4}{|l|}{ B. Sex-cord stromal neoplasm: } \\
\hline Fibrothecoma & 4 cases & $8 \%$ & Unilat \\
\hline Fibroma & 1 case & $2 \%$ & Unilat \\
\hline \multicolumn{4}{|l|}{ C. Germcell neoplasm: } \\
\hline Teratoma & 5 cases & $10 \%$ & 2/5 bilateral \\
\hline Dysgerminoma. & 2 cases & $4 \%$ & $1 / 2$ bilateral \\
\hline D. Metastatic (Krukenberg): & 1 case & $2 \%$ & Bilateral \\
\hline E. Others: & & & $4 / 5$ bilateral \\
\hline Endometrioti cyst & 5 cases & $10 \%$ & 1 non specific. \\
\hline Inflammatory cyst & 2 cases & $4 \%$ & 1 TB granuloma (bilateral) \\
\hline
\end{tabular}


Table 4. Comparison between frozen section and paraffin section according to the status of malignancy.

\begin{tabular}{ccccc}
\hline & Paraffin section & & Total \\
\cline { 2 - 3 } Frozen section & Benign & Borderline & Malignant & \\
Benign & & & & 29 \\
Borderline & 29 & 0 & 0 & 3 \\
Malignant & 0 & 1 & 2 & 18 \\
Total & 29 & 0 & 20 & 50 \\
\hline
\end{tabular}

Table 5. Validity of frozen section in the diagnosis of ovarian masses in relation to parafin section.

\begin{tabular}{cccc}
\hline & Benign & Borderline & Malignant \\
\hline Sensitivity & 100.0 & 100.0 & 90.0 \\
Specificity & 100.0 & 95.9 & 100.0 \\
Positive predictive value & 100.0 & 33.3 & 100.0 \\
Negative predictive value & 100.0 & 100.0 & 93.8 \\
Accuracy & 100.0 & 96.0 & 96.0 \\
\hline
\end{tabular}

\section{Discussion}

Intraoperative frozen section for use in diagnosis of ovarian tumors is of great value. In some cases, it can help surgeons avoid under-treatment or overtreatment of patients. Frozen section is indicated to ensure that the tissue sampled adequate for diagnosis, to determine the nature of the disease process, to plan ancillary studies, to determine tumor spread, and to assess margins [8]. Most studies have shown that if ovarian tumors are categorized according to their malignant potential, the accuracy of frozen section diagnosis would be in the range of $89.8 \%$ $97 \%$ [9] [10].

Baker and Oliva indicated that in some cases a careful gross examination may be as accurate as frozen section and may avoid delays in diagnosis [10]. Given the difficulty in distinguishing borderline from invasive (malignant) tumors, they advocate the use of the term "at least border line" as a reasonable frozen section opinion, which indicates that the final diagnosis may change but allows the surgeon to proceed with a staging operation, which avoids a second surgery [10].

A firm diagnosis of border line neoplasia, however, would allow a less extensive procedure, especially if the patient wished to retain her fertility [11].

Accuracy of frozen section in this era is important to tailor the extent of surgery. This is especially important for young women who has not completed child bearing conservative surgery has been recommended for stage la grade I epithelial ovarian tumors [12] [13]. Also germ cell tumors can be managed by unilateral salpingoopherectomy even if they are in advanced stage as far as these tumors are extremely sensitive to chemotherapy [14] [15].

Suboptimal operation and decreased survival rate will occur in patients with ovarian cancer due to the surgeon's underestimated judgment versus radical surgery and decreased fertility or surgical menopause will be done in patients with ovarian masses due to the surgeon's overestimated judgment [16].

A critical analysis demonstrated a tendency amongst the surgeons to underestimate real neoplasm as benign lesion, probably due to the low statistical likelihood of malignant neoplasm in premenopausal women [16].

Most studies have shown that if ovarian tumors are categorized according to their malignant potential, the accuracy of frozen section diagnosis would be in the range of $89.8 \%$ - 97\% [17]. The overall accuracy in this study was $100 \%$ for benign ovarian neoplasms, $96 \%$ for borderline ovarian neoplasms and $96 \%$ for malignant ovarian neoplasms. In this study the sensitivity for malignant tumors was $90 \%$, while the specificity and positive predictive value was $100 \%$, negative predictive value was $93 \%$, with accuracy of $96 \%$, which is in agreement with the 
literature.

The sensitivity for borderline tumors was $100 \%$, while the specificity was $95 \%$ and positive predictive value was $33 \%$, negative predictive value was $100 \%$, with accuracy of $96 \%$. This not matched with the literatures due to small sample size.

The validity of frozen section as a diagnostic tool of benign tumor was $100 \%$, the sensitivity, the specificity, positive predictive value, negative predictive value, and accuracy for benign tumors were all 100 percentage, which is in agreement with the literature.

In a systematic review of papers examining the use of intraoperative frozen section in predicting the final diagnosis of ovarian lesions by [18], the sensitivity of frozen section was greater than 95\% when benign tumors were compared with borderline and invasive carcinomas but just less than this when borderline and invasive carcinomas were compared. Specificity also exceeded 95\% when benign and invasive carcinoma were compared but was less than this when borderline lesions were compared firstly with benign and secondly with invasive lesions. The positive and negative predictive values were also optimal when benign and invasive carcinomas were compared but reduced when either benign or invasive carcinomas were compared with borderline lesions [18].

According to a meta-analysis of 18 studies, if the diagnosis "borderline" is classified as malignant, the sensitivity of frozen section diagnosis varies between $65 \%$ and $97 \%$, and the specificity between $97 \%$ and $100 \%$ [9].

Ilker et al. analyzed data of 266 patients with ovarian masses. The results of frozen sections were 235 (88.3\%) benign, 11 (4.2\%) borderline and 20 (7.5\%) malignant. The sensitivity was $100 \%$ for the benign tumors, $77.8 \%$ for the borderline tumors and $71.4 \%$ for the malignant tumors. The specificity of frozen section was $83.8 \%$, $98.4 \%$ and $100 \%$ for the benign, borderline and malignant tumors, respectively [19].

In borderline neoplasms, under-diagnosis is generally more frequent than overdiagnosis and the majority of discrepancies also occur in frozen section diagnosis of benign lesions with final report of borderline neoplasms. Even in some series, no borderline tumor was overdiagnosed as invasive cancer [20]. In this study there was an underdiagnosis of 2 malignant cases as borderline by frozen section, this is agreed with the results of literature.

Suprasert et al. recommended that multiple frozen section samples may help increase the accuracy in the diagnosis and taking one frozen section per each $10 \mathrm{~cm}$ diameter of the mass was suggested. However, multiple frozen sections may not be able to eliminate the deferred or discordant cases. In addition, performing many frozen sections in each case is time-consuming and is usually not practical in the intra-operative settings. The deferred diagnostic category reflects an uncertainty in the diagnosis based on limited tissue sampling. An adequate sampling is usually required for a definite diagnosis of such examples as the diagnostic areas may be focal in nature [21].

Houck et al. reviewed 140 cases of borderline tumors, and concluded that only mucinous and mixed histologies versus serous were responsible for underdiagnosis of borderline tumors in frozen sections [20]. Pavlakis et al. assessed 932 ovarian tumors cases and found that the majority of discordant cases had mucinous histology and tumor diameter of more than $10 \mathrm{~cm}$. So they recommended that, care should be taken for large tumors measuring $>20 \mathrm{~cm}$ in diameter, particularly when the intraoperative diagnosis reveals an epithelial borderline tumor or a teratomatous tumor with an extensive neural component [22]. It has also been suggested that, among the common types of borderline tumors, the diagnostic difficulty is greater in the mucinous type compared to the serous counterpart [17] [20]. Large tumor size and tumor heterogeneity of mucinous tumors were the main contributing factors of the diagnostic difficulty.

In the discordant cases, of this study, there were two false positive results (4\%), borderline tumors misinterpreted as "malignant”. Overdiagnosis results in more morbidity especially when the frozen section is diagnosed as malignant. The rate of overdiagnosis in other studies was in the range of the literatures [9].

Metastatic ovarian tumors account for about $6 \%-7 \%$ of ovarian cancer. Differentiating metastatic tumors from primary ovarian cancers is necessary for optimal intraoperative management. Bilaterality, lymphovascular invasion, extensive surface involvement and multinodular pattern of growth are some features that can be used to suggest a metastatic ovarian tumor intraoperatively [23].

In this study, one case is a metastatic tumor and had been identified as "malignant”, but the metastatic nature of tumor had been not identified intraoperatively. The tumor was determined as metastatic in permanent sections, after receiving adequate clinical history and by histologic examination of the specimens received for permanent sections and finally diagnosed as metastatic adenocarcinoma of gastroenterology origin; with extensive intraabdominal spread. In general, frozen section has been reported to be only moderately accurate in differentiating primary versus metastatic malignancy [10]. 
Wootipoom et al. had 16 deferred cases (7\%), consisting primarily of mucinous and stromal tumors [24]. Usubutun et al. also agreed with the present study that mucinous tumors, tumors resembling fibrothecomas, and extensive hemorrhage or necrosis always obscured the frozen section diagnosis. The mean diameter of the mucinous tumors in the deferred group was significantly larger than the mean diameter of the mucinous tumors in the agreement group [25].

Geomini et al. considered deferred results of frozen section as benign tumor because radical surgery should not be performed if malignant diagnosis is not confirmed. The likelihood ratio for malignancy in cases of deferred diagnosis is too small to justify radical surgery [9].

Wootipoom et al. agree with the meta-analysis findings that indicate it is best to wait for final paraffin section results before deciding to perform radical surgery in a case of deferred diagnosis [24].

Wootipoom et al. concluded that intraoperative frozen section diagnosis is generally accurate and can be used as one piece of evidence for the surgeon to use in determining the type and extent of initial surgery to be performed. However, frozen section has limitations such as sampling error, deferred diagnosis and interpretation error. Good intraoperative communication between surgeons and pathologists and regular clinico-pathologic conferences, especially in cases with discordant diagnosis, can maximize accuracy and minimize limitations such as interpretation error and deferred diagnosis [24].

Wootipoom et al. [24] made an effort to improve the frozen section accuracy. They revealed overall accuracy of intraoperative frozen section diagnosis for ovarian tumor ranges between $89.8 \%$ and $97 \%$. After evaluation, they found some limitations of the frozen section diagnosis which needed to be improved such as sampling error, deferred cases, and interpretation error. Sampling error has often been mentioned as one of the limitations of frozen section diagnosis.

According to Stewart et al., several factors can affect the accuracy of frozen section diagnosis, including sampling error, quality of frozen section (technical factor), and experience of pathologists [6].

Sampling error was considered to be the main reason for the diagnostic discrepancy in most studies,. predominantly in some groups of tumors, in which there was remarkable heterogeneity of tumor from areas to areas within the same ovarian mass, such as mucinous tumors and teratomas. Frozen section may fail to sample the most severe lesion or frankly malignant area in a limited number of sections intraoperatively [26].

Another factor that causes difficulty in frozen section diagnosis is the quality of sections prepared by this technique that limits the evaluation of cellular detail. This technical factor is rather underemphasized in most clinical studies on frozen section diagnosis [6]. The section quality was a reason of deferred or discordant diagnoses in this study, also it caused difficulty in some cases of primary epithelial carcinoma in the agreement group with paraffin diagnosis. However, such limitation did not result in the alteration of the intra-operative surgical management in our cases.

With the exception of borderline tumors, the sensitivity and specificity of frozen section diagnosis of ovarian tumors is high. Intraoperative frozen sections for diagnosis of ovarian tumors are of value in preventing the under-treatment and overtreatment of patients when the surgeons and pathologists are aware of limitations. Finally, to reduce the number of false negatives, more extensive frozen section sampling should be performed for clinically suspicious cases in which the speed of diagnosis can be compromised.

Rakhshan et al., concluded that to minimize the discordant cases, one can emphasize on the benefits of the communication between the pathologist and the surgeon about preoperative imaging and intraoperative findings and also a better examination of the larger tumors for appropriate sampling of focal borderline or invasive foci [27].

\section{Conclusion}

In conclusion, intraoperative frozen section is accurate for rapid diagnosis of ovarian tumors. It can help surgeons avoid under-treatment or overtreatment of patients. Our study was designed prospectively using a small number of patients. The door is open to larger studies using a larger number of patients to be performed in order to substantiate our results.

\section{References}

[1] DiSaia, P.J. and Creasman, W.T. (2008) The Adnexal Mass and Early Ovarian Cancer. 7th Edition, Clinical Gynecologic Oncology, Mosby, Chapter 10, 283-312. 
[2] Russell, P., Robboy, S. and Anderson, M. (2002) The Ovary: Normal Appearances and Non-Neoplastic Conditions. In: Robboy, S.J., Anderson, M.C. and Russell, P., Eds., Pathology of the Female Reproductive Tract, Churchill Livingstone, London, 475-526.

[3] Walsh, P. and Cooper, N. (2005) Cancer Ovary Atlas of the United Kingdom and Ireland. 193-201.

[4] Redman, C., Duffy, S., Bromham, N. and Francis, K., NICE, Clinical Guidelines, No. 122. Guideline Development Group (2011) Recognition and Initial Management of Ovarian Cancer: Summary of NICE Guidance. BMJ, 342, d2073.

[5] Khunamornpong, S. and Siriaunkgul, S. (2003) Scrape Cytology of the Ovaries: Potential Role in Intraoperative Consultation of Ovarian Lesions. Diagnostic Cytopathology, 28, 250-257. http://dx.doi.org/10.1002/dc.10273

[6] Stewart, C.J., Brennan, B.A., Hammond, I.G., et al. (2006) Intraoperative Assessment of Ovarian Tumors: A 5-Year Review with Assessment of Discrepant Diagnostic Cases. The International Journal of Gynecological Pathology, 25, 216-222.

[7] Deb, P., Malik, A. and Sinha, K.K. (2011) Intraoperative Scrape Cytology: Adult Granulosa Cell Tumor of Ovary. Journal of Cytology, 28, 207-209. http://dx.doi.org/10.4103/0970-9371.86350

[8] Ilvan, S., Ramazanoglu, R., Akyildiz, E.U., et al. (2005) The Accuracy of Frozen Section (Intraoperative Consultation) in the Diagnosis of Ovarian Masses. Gynecologic Oncology, 97, 395-399. http://dx.doi.org/10.1016/j.ygyno.2005.01.037

[9] Geomini, P., Bremer, G., Kruitwagen, R., et al. (2005) Diagnostic Accuracy of Frozen Section Diagnosis of the Adnexal Mass: A Metaanalysis. Gynecologic Oncology, 96, 1-9. http://dx.doi.org/10.1016/j.ygyno.2004.09.042

[10] Baker, P. and Oliva, E. (2008) A Practical Approach to Intraoperative Consultation in Gynecological Pathology. The International Journal of Gynecological Pathology, 27, 353-365.

[11] Medeiros, L.R., Rosa, D.D., Edelweiss, M.I., Stein, A.T., Bozzetti, M.C., Zelmanowicz, A., et al. (2005) Accuracy of Frozen-Section Analysis in the Diagnosis of Ovarian Tumors: A Systematic Quantitative Review. International Journal of Gynecological Cancer, 15, 192-202. http://dx.doi.org/10.1111/j.1525-1438.2005.15203.X

[12] Morice, P., Camatte, S., El Hassan, J., Pautier, P., Duvillard, P. and Castaigne, D. (2001) Clinical Outcomes and Fertility after Conservative Treatment of Ovarian Borderline Tumors. Fertility and Sterility, 75, 92-96. http://dx.doi.org/10.1016/S0015-0282(00)01633-2

[13] Morice, P., Camatte, S., Wickart-Poque, F., Rouzier, R., Pautier, P., Pomel, C., et al. (2002) Conservative Management of Malignant and Borderline Ovarian Tumor. Bulletin du Cancer, 89, 1019-1026.

[14] Moodley, M., Moodley, J. and Chikosi, A.B. (2000) Ovarian Carcinoma, Pericardial Metastasis and Human Immunodeficiency Virus Infection. International Journal of Gynecological Cancer, 10, 82-83. http://dx.doi.org/10.1046/j.1525-1438.2000.00008.x

[15] Molina, D. and Hurteau, J. (2002) Ovarian Germ Cell Tumors. Current Problems in Obstetrics, Gynecology and Fertility, 25, 230-236.

[16] Ghaemmaghami, F., Fakour, F., Zarchi, M.K., Behtash, N., Gilani, M.M., Mousavi, A., et al. (2008) Clinical Assessment, Gross Examination, Frozen Section of Ovarian Masses: Do Patients Benefit? Archives of Gynecology and Obstetrics, 278, 209-213. http://dx.doi.org/10.1007/s00404-007-0553-8

[17] Tempfer, C.B., Polterauer, S., Bentz, E.K., Reinthaller, A. and Hefler, L.A. (2007) Accuracy of Intraoperative Frozen Section Analysis in Borderline Tumors of the Ovary: A Retrospective Analysis of 96 Cases and Review of the Literature. Gynecologic Oncology, 107, 248-252. http://dx.doi.org/10.1016/j.ygyno.2007.06.008

[18] Heatley, M.K. (2012) A Systematic Review of Papers Examining the Use of Intraoperative Frozen Section in Predicting the Final Diagnosis of Ovarian Lesions. International Journal of Gynecological Pathology, 31, 111-115. http://dx.doi.org/10.1097/PGP.0b013e318226043b

[19] Ilker, A., Aykut, B., Muge, H., Ibrahim, H.M., Ulku, O.B., Sener, G., et al. (2011) Accuracy of Intra-Operative Frozen Section in the Diagnosis of Ovarian Tumours. Journal of Pakistan Medical Association, 61, 856-858.

[20] Houck, K., Nikrui, N., Duska, L., Chang, Y., Fuller, A.F., Bell, D., et al. (2000) Borderline Tumors of the Ovary: Correlation of Frozen and Permanent Histopathologic Diagnosis. Obstetrics \& Gynecology, 95, 839-843. http://dx.doi.org/10.1016/S0029-7844(99)00656-0

[21] Suprasert, P., Khunamornpong, S., Phusong, A., Settakorn, J. and Siriaungkul, S. (2008) Accuracy of Intra-Operative Frozen Sections in the Diagnosis of Ovarian Masses. Asian Pacific Journal of Cancer Prevention, 9, 737-740.

[22] Pavlakis, K., Messini, I., Vrekoussis, T., Yiannou, P., Panoskaltsis, T. and Voulgaris, Z. (2009) Intraoperative Assessment of Epithelial and Non-Epithelial Ovarian Tumors: A 7-Year Review. European Journal of Gynaecological Oncology, 30, 657-660.

[23] Tangjitgamol, S., Jesadapatrakul, S., Manusirivithaya, S. and Sheanakul, C. (2004) Accuracy of Frozen Section in Diagnosis of Ovarian Mass. International Journal of Gynecological Cancer, 14, 212-219. 
http://dx.doi.org/10.1111/j.1048-891X.2004.014202.x

[24] Wootipoom, V., Dechsukhum, C., Hanprasertpong, J., et al. (2006) Accuracy of Intraopera-Tive Frozen Section in Diagnosis of Ovarian Tumors. Journal of the Medical Association of Thailand, 89, 577-582.

[25] Usubutun, A., Altinok, G. and Kucukali, T. (1998) The Value of Intraoperative Consultation (Frozen Section) in the Diagnosis of Ovarian Neoplasms. Acta Obstetricia et Gynecologica Scandinavica, 77, 1013-1016.

[26] Wasinghon, P., Suthippintawong, C. and Tuipae, S. (2008) The Accuracy of Intraoperative Frozen Sections in the Diagnosis of Ovarian Tumors. Journal of the Medical Association of Thailand, 91, 1791-1795.

[27] Rakhshan, A., Zham, H. and Kazempour, M. (2009) Accuracy of Frozen Section Diagnosis in Ovarian Masses: Experience at a Tertiary Oncology Center. Archives of Gynecology and Obstetrics, 280, 223-228.

http://dx.doi.org/10.1007/s00404-008-0899-6 
Scientific Research Publishing (SCIRP) is one of the largest Open Access journal publishers. It is currently publishing more than 200 open access, online, peer-reviewed journals covering a wide range of academic disciplines. SCIRP serves the worldwide academic communities and contributes to the progress and application of science with its publication.

Other selected journals from SCIRP are listed as below. Submit your manuscript to us via either submit@scirp.org or Online Submission Portal.
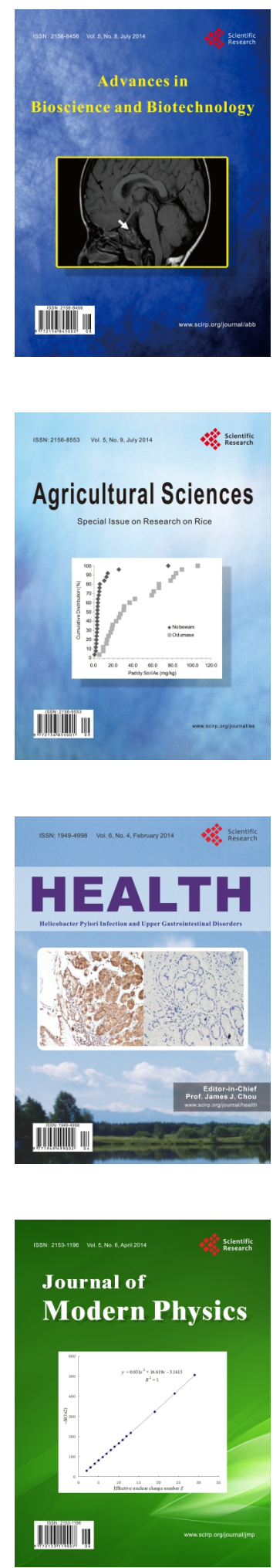
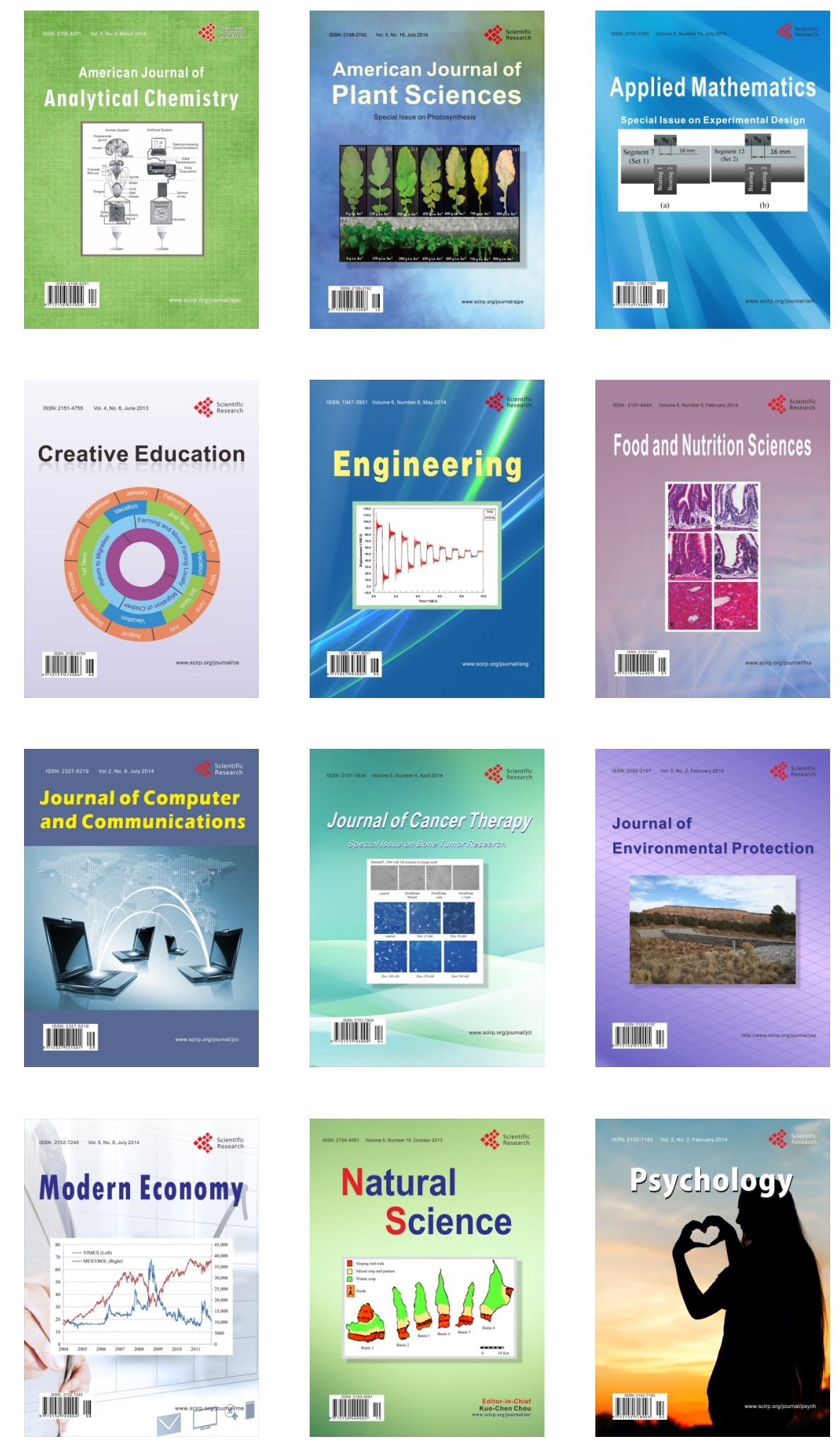